\title{
Retinal Counterion Switch Mechanism in Vision Evaluated by Molecular Simulations
}

\author{
Karina Martínez-Mayorga, ${ }^{\dagger}$ Michael C. Pitman, ${ }^{\ddagger}$ Alan Grossfield, ${ }^{\ddagger}$ Scott E. Feller,$\S$ and \\ Michael F. Brown ${ }^{*},+, \perp$ \\ Departments of Chemistry and Physics, University of Arizona, Tucson, Arizona 85721, IBM T. J. Watson Research \\ Center, Yorktown Heights, New York 10598, and Department of Chemistry, Wabash College, \\ Crawfordsville, Indiana 47933
}

Received October 7, 2006; E-mail: mfbrown@u.arizona.edu

G protein-coupled receptors (GPCRs) comprise the largest protein family in the human genome, ${ }^{1}$ yet rhodopsin is the only member for which there is a high-resolution crystal structure. ${ }^{2}$ Understanding GPCR activation is significant due to its role in drug discovery and development. Of ca. 500 molecular sites for current drug therapy, cellular membrane receptors are the largest subgroup and represent $45 \%$ of all targets. ${ }^{3}$ Here we show how analysis of the rhodopsin chromophore retinal by molecular dynamics (MD) simulations with experimental ${ }^{2} \mathrm{H}$ NMR data gives insights into the counterion switch mechanism that stabilizes the protonated Schiff base (PSB). Disruption of the retinal PSB is thermodynamically the most important event that characterizes the activated metarhodopsin II (MII) state. ${ }^{4}$

Current advances in supercomputing ${ }^{5}$ have made it possible to extend MD simulations to the unprecedented time scales described here. The strength of MD simulations is that they explore the structure and fluctuations of membrane proteins with atomic-level resolution. Such models are the culmination of extensive methods development, where force fields with rigorous inclusion of longrange electrostatics are validated by careful comparison with experimental data. ${ }^{5,6}$ We performed two simulations of a system composed of one rhodopsin (Rho) molecule embedded in a lipid bilayer of 1-stearoyl-2-docosahexaenoyl-sn-glycero-3-phosphocholine (SDPC), 1-stearoyl-2-docosahexaenoyl-sn-glycero-3-phosphoethanolamine (SDPE), and cholesterol (49:50:24) hydrated by 7400 waters, for 43222 atoms in total. The simulations were run for 1500 and 2000 ns after flash isomerization ${ }^{6}$ and corresponded to metarhodopsin I (MI) formation (cf. Supporting Information, SI). The primary difference between the simulations was the initial protonation state of the Glu181 residue.

Insight into the counterion switch in MI formation is vital to understanding the subsequent activated MII state. ${ }^{4}$ Atomic-level structures are unavailable for MI and MII, though a structure exists for MI at low resolution from electron crystallography. ${ }^{7}$ As a result, counterion switch models are largely based on vibrational (resonance Raman and FTIR) and UV-visible spectroscopy of sitedirected mutants of Rho. ${ }^{8,9}$ However, classical MD simulations can only be directly compared with such results under very specific circumstances. According to the original proposal, ${ }^{8}$ in the dark and bathorhodopsin states, the retinal PSB is stabilized by the carboxylate of Glu113 in transmembrane helix H3, whereas Glu181 is protonated. Hence the Rho binding pocket is neutral (Scheme 1). After retinal isomerization, a proton transfer (tautomerization) from Glu181 to Glu113 takes place as the protein enters the lumirhodopsin state. Repositioning of retinal within its binding pocket in the

\footnotetext{
$\dagger$ Department of Chemistry, University of Arizon.

${ }^{\perp}$ Department of Physics, University of Arizona.

¥ IBM T. J. Watson Research Center.

$\S$ Wabash College.
}

\section{Scheme 1}

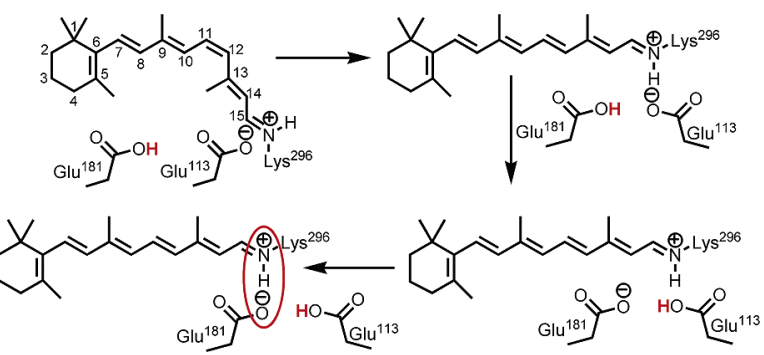

Scheme 2

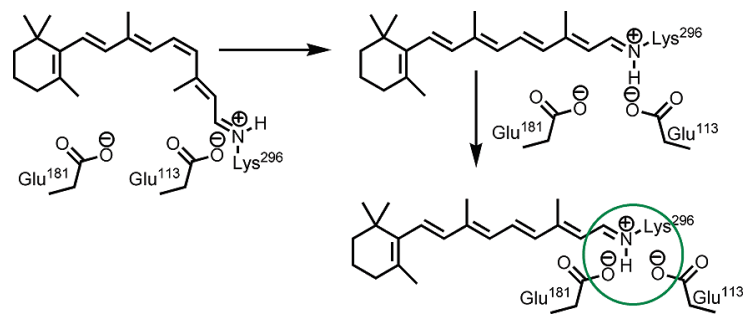

MI state then switches the counterion from Glu113 in helix $\mathrm{H} 3$ to Glu181 in the E2 extracellular plug of Rho, which may be a general mechanism of receptor activation. ${ }^{8}$

Recently, an alternative complex-counterion switch model ${ }^{9}$ has been proposed, in which both Glu113 and Glu181 exist as glutamates in the dark state and remain deprotonated through the MI state, giving a net negative charge to the binding pocket. In this mechanism, Glu113 stabilizes the PSB together with the hydrogen-bonded network involving the extracellular loop E2 until the lumirhodopsin state is reached (Scheme 2).${ }^{10}$ Reorientation of retinal in MI leads to a shift of the counterion function, predominantly from Glu113 in the dark to Glu181 in the MI state. Thereafter, deprotonation of the retinylidene Schiff base occurs in the activated MII state, leading to destabilization of the salt bridge and receptor activation.

The simulation for Scheme 1 began with Glu181 protonated (neutral) and Glu113 charged; the proton was explicitly shifted from Glu181 to Glu113 500 ns after the retinal isomerization. For Scheme 2 , both glutamates were charged throughout the simulation. Upon isomerization, the polyene chain became more planar than the conformationally distorted dark state. ${ }^{11}$ For Scheme 1, our conclusion that the MI state was reached involved a switch in the distances of Glu113 and Glu181 to the PSB, which was stable for $\sim 1000-$ 2000 ns; the last 500 ns were designated as MI. We calculated the angle between the $\mathrm{C}-\mathrm{CH}_{3}$ bond vector versus the membrane normal (z-axis) for each of the methyl groups to test the simulation predictions against experimental ${ }^{2} \mathrm{H}$ NMR data, which gave detailed information on the orientation of specific bonds in the retinal (see 


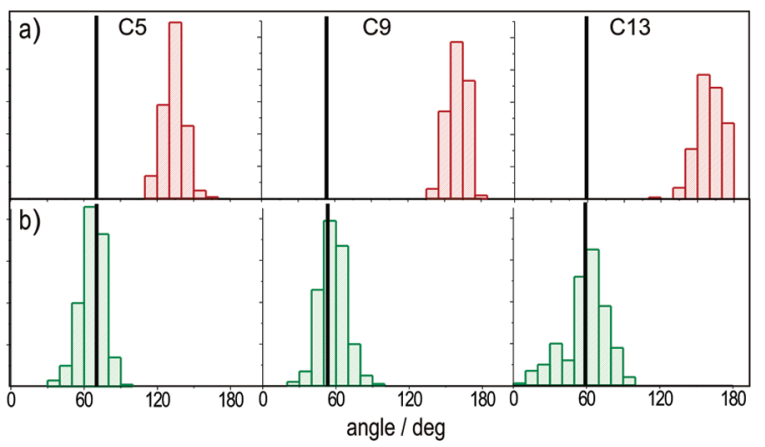

Figure 1. Distribution of bond orientations of $\mathrm{C} 5, \mathrm{C} 9$, and $\mathrm{C} 13$ methyl groups of retinal in MI structure derived for (a) counterion switch and (b) complex-counterion switch models. Filled black rectangles correspond to experimental ${ }^{2} \mathrm{H}$ NMR spectra. ${ }^{12}$

SI for details). In Figure 1a, the bond distributions for Scheme 1 show that the methyl groups fluctuate in a narrow range $\left(20^{\circ}\right)$, with a mean orientation $\sim 130^{\circ}$ for $\mathrm{C} 5$ and $160^{\circ}$ for the C9 and C13 methyls. However, these values disagree with experimentally derived bond orientations from ${ }^{2} \mathrm{H}$ NMR spectroscopy. For Scheme 2, a switch in the distance from Glu113 and Glu181 was also seen, which was stable from 1000 to $1500 \mathrm{~ns}$, with larger amplitude fluctuations. The MI state was considered formed after $1000 \mathrm{~ns}$ of simulation. In Figure 1b, the bond angle distributions for Scheme 2 are broader, especially for the $\mathrm{C} 13$ methyl, with mean orientations of $\sim 50-60^{\circ}$. More importantly, these complex-counterion simulations show essentially perfect agreement with the experimental ${ }^{2} \mathrm{H}$ NMR results. This is particularly striking given there are no adjustable parameters whatsoever in this comparison, and that the NMR data do not enter into construction of the model. The predictive power of the simulations is further validated by the observation that the MI structures are different from each other and from the initial (dark state) conditions, indicating that sufficient phase space is explored during the microsecond scale simulations.

In order to connect more directly to the experimental ${ }^{2} \mathrm{H} \mathrm{NMR}$ data, we also calculated ${ }^{13}$ theoretical spectra for the $\mathrm{C} 5, \mathrm{C} 9$, and C13 methyl groups. Bond orientational distributions (Figure 1) from the MD simulations were used to weight the theoretical line shapes computed with a Monte Carlo method for a static uniaxial distribution with mosaic spread. ${ }^{13}$ Simulated spectra are shown in Figure 2 versus the experimental solid-state ${ }^{2} \mathrm{H}$ NMR data. ${ }^{12}$ For each methyl group, we display three representative orientations of $\theta=0,45$, and $90^{\circ}$ for the bilayer normal relative to the magnetic field $\mathbf{B}_{\mathbf{0}}$. Figure $2 \mathrm{a}$ shows that the counterion switch simulation does not agree with the ${ }^{2} \mathrm{H}$ NMR data, as is most evident for the $0^{\circ}$ tilt angle. By contrast, the complex-counterion model shows excellent agreement with the ${ }^{2} \mathrm{H}$ NMR spectra of retinal within the binding cavity of rhodopsin at all tilt angles (Figure 2b).

The origin of the different structures is the negative charge in the Rho binding pocket in the complex-counterion switch mechanism. For either simulation, the protonation state of Glu181 leads to a counterion switch. In Scheme 2, the retinal orientation is closer to the dark state than in the counterion switch model and corresponds to electron crystallography data that show the position of retinal in MI remains similar to that in the dark state. ${ }^{7}$ The X-ray structure of lumirhodopsin recently obtained at $2.7 \AA$ resolution ${ }^{14}$ also shows that the retinal position is close to the dark state. Thus both the crystallographic and NMR data lead us to conclude that Scheme 2 best describes the pathway for MI formation. On the basis of the $\mathrm{C} 5, \mathrm{C} 9$, and $\mathrm{C} 13$ bond orientations of retinal, we suggest that the protonation state of the Rho binding pocket controls the relaxation of retinal to reach the preactive MI state.

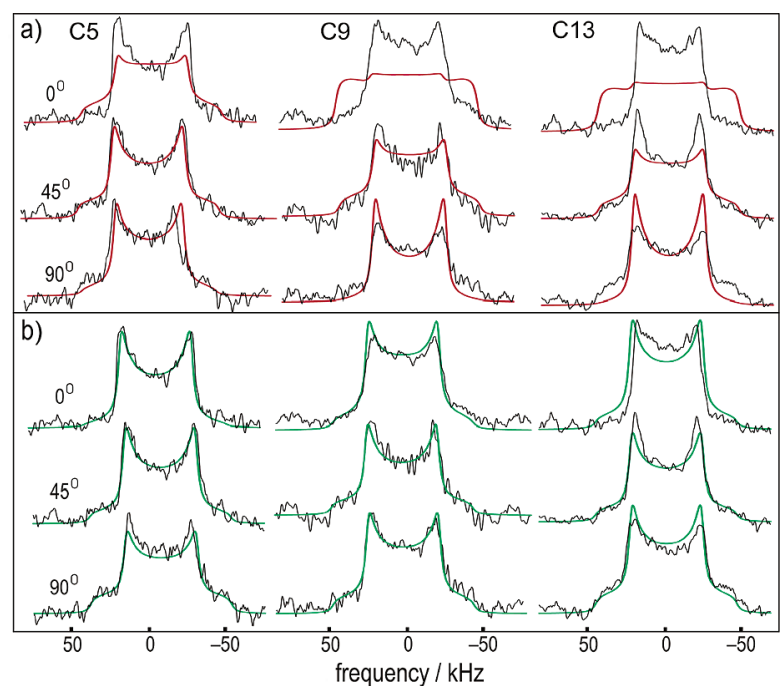

Figure 2. Experimental solid-state ${ }^{2} \mathrm{H}$ NMR spectra for $\mathrm{C} 5$, $\mathrm{C} 9$, and $\mathrm{C} 13$ ${ }^{2} \mathrm{H}$-labeled methyl groups of retinal in MI state at $-100{ }^{\circ} \mathrm{C}$ at 0,45 , and $90^{\circ}$ tilt angles. ${ }^{12}$ Continuous color lines are based on MD simulations: (a) counterion switch model and (b) complex-counterion switch model.

Our work shows that very long time scale MD simulations are able to predict ${ }^{2} \mathrm{H}$ NMR spectra for the orientation of the retinylidene chromophore of rhodopsin. Further, they enable distinction between counterion mechanisms in MI formation. Extension of this approach to MII can yield insight into the mechanism of rhodopsin activation in visual signaling.

Acknowledgment. We thank P.-W. Lau, T. Sakmar, and A. Struts for discussions. Work was supported by NSF (S.E.F.), NIH (M.F.B.), and IBM Watson BGW Blue Gene facility.

Supporting Information Available: Modeling system construction. This material is available free of charge via the Internet at http:// pubs.acs.org.

\section{References}

(1) Fanelli, F.; De Benedetti, P. G. Chem. Rev. 2005, 105, 3297-3351.

(2) (a) Palczewski, K.; Kumasaka, T.; Hori, T.; Behnke, C. A.; Motoshima, H.; Fox, B. A.; Le Trong, I.; Teller, D. C.; Okada, T.; Stenkamp, R. E.; Yamamoto, M.; Miyano, M. Science 2000, 289, 739-745. (b) Okada, T.; Sugihara, M.; Bondar, A.-N.; Elstner, M.; Entel, P.; Buss, V. J. Mol. Biol. 2004, 342, 571-583.

(3) (a) Drews, J. Science 2000, 287, 1960-1964. (b) Filmore, D. Modern Drug Discovery 2004, 7, 24-28.

(4) (a) Sakmar, T. P.; Menon, S. T.; Marin, E. P.; Awad, E. S. Annu. Rev. Biophys. Biomol. Struct. 2002, 31, 443-484. (b) Hubbell, W. L.; Altenbach, C.; Hubbell, C. M.; Khorana, H. G. Adv. Protein Chem. 2003, 63, 243-290.

(5) (a) Pitman, M. C.; Grossfield, A.; Feller, S. E. J. Am. Chem. Soc. 2005 127, 4576-4577. (b) Grossfield, A.; Feller, S. E.; Pitman, M. C. Proc. Natl. Acad. Sci. U.S.A. 2006, 103, 4888-4893.

(6) Saam, J.; Tajkhorshid, E.; Hayashi, S.; Schulten, K. Biophys. J. 2002 83, 3097-3112

(7) Ruprecht, J. J.; Mielke, T.; Vogel, R.; Villa, C.; Schertler, G. F. X. EMBO J. 2004, 23, 3609-3620.

(8) Yan, E. C. Y.; Kazmi, M. A.; Ganim, Z.; Hou, J.-M.; Pan, D.; Chang, B. S. W.; Sakmar, T. P.; Mathies, R. A. Proc. Natl. Acad. Sci. U.S.A. 2003 100, 9262-9267.

(9) Lüdeke, S.; Beck, M.; Yan, E. C. Y.; Sakmar, T. P.; Siebert, F.; Vogel, R. J. Mol. Biol. 2005, 353, 345-356.

(10) Vogel, R.; Siebert, F.; Yan, E. C. Y.; Sakmar, T. P.; Hirshfeld, A.; Sheves, M. J. Am. Chem. Soc. 2006, 128, 10503-10512.

(11) Salgado, G. F. J.; Struts, A. V.; Tanaka, K.; Fujioka, N.; Nakanishi, K.; Brown, M. F. Biochemistry 2004, 43, 12819-12828.

(12) Salgado, G. F. J.; Struts, A. V.; Tanaka, K.; Krane, S.; Nakanishi, K.; Brown, M. F. J. Am. Chem. Soc. 2006, 128, 11067-11071.

(13) Nevzorov, A. A.; Moltke, S.; Heyn, M. P.; Brown, M. F. J. Am. Chem Soc. 1999, 121, 7636-7643.

(14) Nakamichi, H.; Okada, T. Proc. Natl. Acad. Sci. U.S.A. 2006, 103, $12729-12734$

JA0671971 